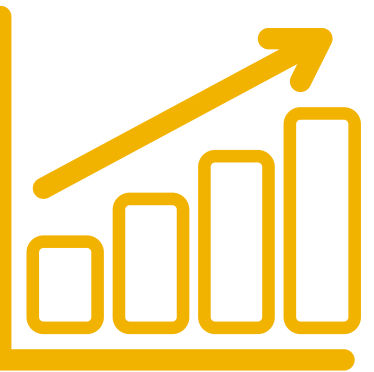

\title{
METODOLOGIAS ATIVAS UTILIZADAS POR PRECEPTORES NAS RESIDÊNCIAS MULTIPROFISSIONAIS EM SAÚDE: SCOPING REVIEW
}

\author{
ACTIVE METHODOLOGIES USED BY PRECEPTORS IN MULTIPROFESSIONAL \\ RESIDENCES: SCOPING REVIEW
}

\begin{abstract}
Sheila Duarte de Mendonça Fernandes
Enfermeira, Mestranda Profissional em Ensino na Saúde da Universidade Federal do Rio Grande do Norte.

E-mail: sheilhinha@gmail.com.

Ágata Pereira Trindade

Discente do Curso Superior de Tecnologia em Gestão Hospitalar da Universidade Federal do Rio Grande do Norte. E-mail: agatatrindade21@gmail.com.

Tayana Cabral Figueiredo

Discente do Curso Superior de Tecnologia em Gestão Hospitalar da Universidade Federal do Rio Grande do Norte. E-mail: tayanacf@gmail.com.

Fernanda Caroline Camara da Costa

Discente do Curso Superior de Tecnologia em Gestão Hospitalar da Universidade Federal do Rio Grande do Norte. E-mail: krol_fccc@hotmail.com.

Samylla Beatriz Régio de Oliveira

Discente do Curso Superior de Tecnologia em Gestão Hospitalar da Universidade Federal do Rio Grande do Norte. E-mail: samyllabeatrizoliveira2018@gmail.com.

Pétala Tuani Candido de Oliveira Salvador

Enfermeira, Doutora em Enfermagem; Professora da Escola de Saúde e do Programa de Pós-Graduação em Ensino na Saúde da Universidade Federal do Rio Grande do Norte. E-mail: petalatuani@hotmail.com.
\end{abstract}

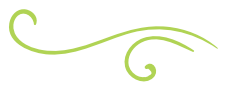

\section{RESUMO}

Os preceptores das Residências Multiprofissionais em Saúde são desafiados a utilizar as estratégias de ensino-aprendizagem mais didáticas e a se distanciar da perspectiva tradicional, como é o caso das metodologias ativas. O objetivo deste estudo é identificar e mapear o uso de metodologias ativas pelos preceptores nas Residências Multiprofissionais em Saúde.
Trata-se de scoping review, que incluiu artigos, teses e dissertações, identificados a partir de 12 bases de dados. A análise se deu por estatística descritiva simples. A amostra final constituiu-se de 10 pesquisas, com predominância de dissertações (50\%). Observou-se publicação ascendente ao longo do tempo, com predominância de estudos no Brasil (90\%) e da área de Enfermagem (30\%). Foi identificado o uso 
de 14 metodologias ativas, com destaque para Tenda invertida (3), Projeto Terapêutico Singular (3), Roda de núcleo (2), Roda de campo (2) e Educação interprofissional (2). As metodologias ativas de ensino têm sido utilizadas pelos preceptores nas Residências Multiprofissionais de Saúde como método de ensino para promover um processo de ensino-aprendizagem ativo e colaborativo dos profissionais em saúde.

Palavras-chave: Residências Multiprofissionais em Saúde. Ensino. Preceptoria.

\section{ABSTRACT \\ Multiprofessional Health Residency preceptors are challenged to use the most didactic teaching and learning strategies and distance themselves from the traditional perspective, as is the case with active methodologies. The objective is to identify and map the use of active methodologies by preceptors in Multiprofessional Health Residencies. This is a scoping review that included articles, theses and dissertations were analyzed, identified from 12 databases. The analysis was performed using simple descriptive statistics. The final sample consisted of 10 surveys, with a predominance of dissertations (50\%). It was observed that the publications had a greater rise during the time, with a predominance of studies in Brazil (90\%) and from Nursing (30\%). The use of 14 active methodologies was identified, with emphasis onlnverted tent (3), Singular Therapeutic Project (3), Core wheel (2), Field wheel (2) and Interprofessional education (2). At active teaching methodologies have been used by preceptors in Multiprofessional Health Residencies as a teaching method to ensure a better teaching-learning process for health professionals.}

Key-words: Multiprofessional Health Residencies. Teaching. Preceptorship.

\section{INTRODUÇÃO}

De acordo com o Ministério da Educação brasileiro, as Residências Multiprofissionais em Saúde (RMS) surgiram no Brasil em 1978, por intermédio da Secretaria Estadual de Saúde do Rio Grande do Sul (BRASIL, 2018). No decorrer do tempo, ocorreram muitas mudanças no contexto da saúde brasileira, como a Reforma Sanitária; a Constituição Brasileira, com a criação do Sistema Único de Saúde (SUS); e a Lei Nº 8.080/90, que o regulamentou. Somente em 2005 foi promulgada a Lei $N^{\circ} 11.129$, que instituiu a Comissão Nacional de Residência Multiprofissional em Saúde (CNRMS) (BRASIL, 2006).

No contexto brasileiro, as RMS ganharam novo significado a partir da criação do SUS, quando se vislumbrou a necessidade de formar profissionais para uma atuação diferenciada, uma vez que o SUS pressupõe a construção interdisciplinar dos profissionais em saúde, trabalho em equipe, dispositivo de educação permanente e reorientação das lógicas técnicas e assistenciais (SILVA et al., 2016). Dessa forma, a RMS se configura como um espaço para o desenvolvimento das ações de Educação Permanente em Saúde (EPS), sendo um dispositivo potencial para a promoção das mudanças pretendidas pelos profissionais de saúde, a fim de consolidar os princípios do SUS (SILVA et al., 2016). Esse modelo, de nível de formação pós-graduada lato sensu conta com o acompanhamento pedagógico de um preceptor, o qual atua como educador e mediador, fortalecendo o trabalho interdisciplinar, interprofissional, fomentando a transversalidade e a grupalidade (LORENZ, 2010).

Além disso, nas RMS, os preceptores são desafiados a conduzir uma prática profissional que ultrapasse um fazer repetitivo e valorize um saber compartilhado, consciente, reflexivo, problematizador e transformador, bem como a desenvolver estratégias de ensino-aprendizagem que promovam um processo educacional capaz de reconstruir novos saberes e gerar corresponsabilização, 
como é o caso das metodologias ativas, com o intuito de deixar o processo de ensino-aprendizagem mais didático e se distanciar da perspectiva tradicional (MATTOS, 2016).

Desse modo, o plano pedagógico das RMS deve prever a utilização de metodologias ativas de ensino, as quais buscam valorizar o vínculo entre o preceptor e o aluno, permitindo que ambos participem do processo de ensino-aprendizagem. Esses métodos estimulam a criticidade e a reflexão dos profissionais, possibilitando que estejam capacitados para todos os desafios que enfrentam nas suas atividades laborais, desse modo, facilita-se o processo de formação (BERBEL, 2011; ROMAN et al., 2017).

Em contraponto, entende-se que $\mathrm{O}$ uso de metodologias ativas pelo preceptor é um desafio, visto que, em geral, ele não dispõe de formação na área pedagógica, contando apenas com seus conhecimentos teóricos a respeito da sua formação na área da saúde. Por consequência, tal fato pode vir a ser uma objeção no processo de ensino-aprendizagem e, nesse contexto, o uso das metodologias ativas de ensino pode constituir uma alternativa positiva e propícia.

Diante disso, compreende-se que esta pesquisa é de suma importância para identificar como as metodologias ativas vêm sendo incorporadas nas RMS, se são eficazes e eficientes para o aprendizado satisfatório dos profissionais, permitindo um entendimento amplo dos processos de trabalho que perpassam esse nível educacional. Acredita-se que é fundamental pensar em metodologias ativas nas RMS, uma vez que os educandos estão inseridos em uma formação que agrega saberes de todos os núcleos profissionais (SALVADOR et al., 2011).

Dessa forma, busca-se responder à seguinte questão de pesquisa: como as metodologias ativas de ensino têm sido utilizadas pelos preceptores nas Residências Multiprofissionais de Saúde? Objetiva-se, com isso, identificar e mapear o uso de metodologias ativas pelos preceptores nas Residências Multiprofissionais em Saúde.

\section{MÉTODO}

Trata-se de scoping review, guiada pelas recomendações do JBI Institute Reviewer's Manual (2015), segundo quadro teórico proposto por Arksey e O'Malley (2005), e apresentada segundo as recomendações do Preferred Reporting Items for Systematic reviews and Meta-Analyses estension for Scoping Reviews (PRISMA-SrR): Checklist and Explanation, seu protocolo foi registrado no Open Science Framework (TRICCO et al., 2018).

Adotou-se um protocolo de pesquisa constituído pelos itens: tipo de estudo, objetivo, composição da amostra, formulação de pergunta, critérios de inclusão, critérios de exclusão, coleta de dados, extração dos dados e síntese de dados. A população de estudo constitui-se de pesquisas produzidas no âmbito de RMS que tenham utilizado metodologias ativas de ensino pelos preceptores.

Em detrimento disso, para a formulação da pergunta de pesquisa, utilizou-se a estratégia PCC (P- Population; C- Concept; C- Context), na qual foi estabelecida como P: Preceptores; C: Metodologias ativas de ensino; C: Residência Multiprofissional em Saúde. Nesse sentido, a seguinte pergunta orientou a scoping review: como as metodologias ativas de ensino têm sido utilizadas pelos preceptores nas Residências Multiprofissionais de Saúde?

A princípio, realizou-se uma busca preliminar nas bases de dados JBI COnNECT+, $D A R E$, The Cochrane Library e PROSPERO e não foram identificados protocolos e revisões com temática semelhante. Dando prosseguimento à pesquisa, foi realizada uma busca no portal Biblioteca Virtual em Saúde (BVS) e U.S. National Library of Medicine (PubMed) para identificar os principais descritores e palavras-chave utilizados nos estudos que abordassem a temática de interesse a partir da combinação dos Descritores em Ciências da Saúde (DeCS) e Medical Subject Headings (MeSH) identificados para o mneumônico da pesquisa: (Preceptorship) AND 
(Problem-Based Learning) AND (Internship, Nonmedical). Dessa forma, foram identificadas as palavras-chave para cada tópico da estratégia PCC.

Logo, a estratégia de busca final para - DeCS e MeSH foi definida: DeCS: (Preceptoria OR Preceptor OR preceptores $O R$ tutores $O R$ tutor $O R$ tutoria $O R$ preceptoras OR preceptora) AND (Aprendizagem Baseada em Problemas OR Aprendizado ativo OR Aprendizado Baseado em Problemas $O R$ Aprendizagem Ativa $O R$ Estratégias de Aprendizagem Ativas $O R$ Metodologia ativa $O R$ Metodologias ativas $O R$ Metodologia centrada no aluno $O R$ Método ativo OR Métodos ativos) AND (Internato não médico $O R$ Residência não Médica não Odontológica $O R$ Residência Multiprofissional OR Residência em Saúde OR Formação multiprofissional em saúde $O R$ Formação de residentes multiprofissionais em saúde OR Residência Integrada em Saúde OR Programa de Residência Integrada Multiprofissional em Saúde $O R$ Residências Multiprofissionais).

MeSH: (Preceptorship OR preceptors OR preceptor OR precepting) AND ("ProblemBased Learning" OR "deep learning" OR "teaching methods" $O R$ "case based learning" OR "methods learner centered" $O R$ "interactive teaching methods" OR "learning-based methods") AND ("Internship, Nonmedical" OR "residency program" OR "postgraduate hospital-based residency program" OR "postgraduatein-hospital residency program" OR "Non medical Residency" OR "residency programs").

Em setembro de 2019, prosseguiu-se com a coleta de dados nas bases PubMed, Cumulative Index to Nursing \& Allied Health Literature (CINAHL), Web of science, Literatura Latino-americana e do Caribe em Ciências da Saúde (LILACS) e Educational Resources Information Center (ERIC). Além disso, foi inserida a literatura cinzenta, a partir do Catálogo de Teses e Dissertações da Coordenação de Aperfeiçoamento de Pessoal de Nível Superior (CAPES),
Academic Archive Online (DIVA), Europe E-theses Portal (DART), Eletronic Theses Online Service (EThOS), Repositório Científico de Acesso Aberto de Portugal (RCAAP), South African National Theses and Dissertations (ETD Portal) e Theses Canada.

Foram incluídas pesquisas de abordagem quantitativa e qualitativa; publicadas na íntegra na língua portuguesa, espanhola, inglesa ou francesa. Foram excluídos editoriais, ensaios teóricos e revisões integrativas e pesquisas que utilizassem outros mecanismos de metodologias de ensino. Não foi delimitado limite temporal, no entanto, na base de dados da CAPES, identificaram-se limitações temporais, pois as publicações estavam disponíveis na íntegra apenas a partir de 2013.

Durante a busca nas bases de dados, foi realizada a análise do título e do resumo de todos os estudos identificados, com base nos critérios de inclusão e exclusão estabelecidos. Logo após, realizou-se o download das publicações pré-selecionadas, identificou-se duplicação de estudos selecionados entre as bases e iniciou-se a extração de dados a partir de planilha construída no Microsoft Excel 2010 das publicações componentes da amostra final.

Procedeu-se à etapa da leitura na íntegra, na qual 34 estudos foram excluídos por não abordar a temática sobre aplicação das metodologias ativas por preceptores, oito por não se aplicar em RMS e cinco por não detalhar o processo de uso das metodologias ativas. Como resultado, dez pesquisas compuseram a amostra final e a planilha de avaliação foi composta pelas variáveis: tipo de estudo (se artigo, dissertação ou tese); ano de publicação; país de origem; formação do autor; tipo de metodologia utilizada; detalhamento do uso da metodologia; potencialidade e desafios do uso das metodologias ativas. A análise foi realizada por quatro pesquisadoras de forma independente. Os dados foram sintetizados de forma descritiva simples. Não foi necessária apreciação ética da pesquisa por se tratarem de dados de domínio público. 


\section{RESULTADOS}

A amostra final foi constituída por 10 estudos, o que representa $0,2 \%$ do número inicial de estudos encontrados e $17,5 \%$ das pesquisas pré-selecionadas para leitura na íntegra (Figura 1).

Figura 1 - Fluxograma PRISMA ScR (adaptado) do processo de seleção do estudo.

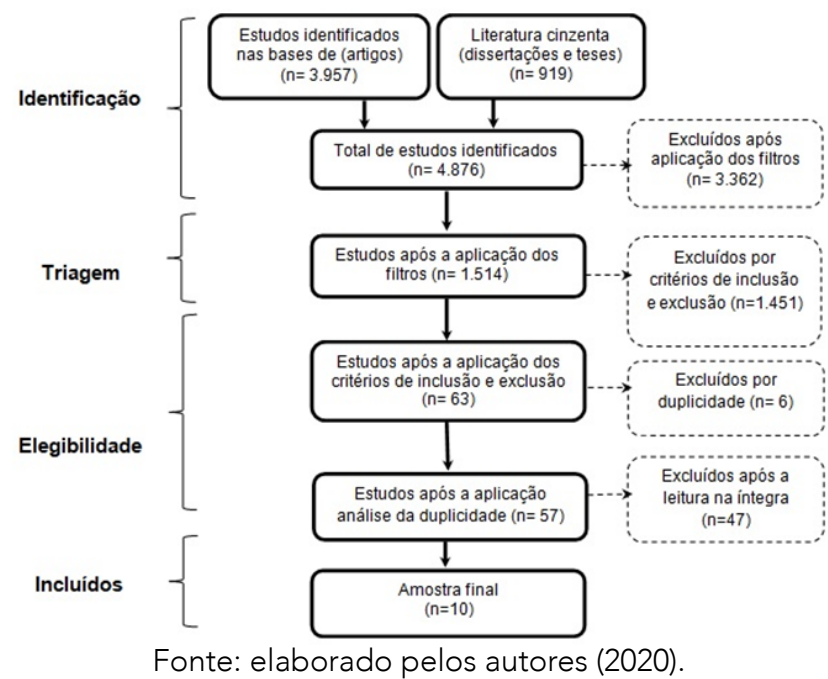

A amostra foi composta, em sua maioria, por dissertações $(5 ; 50 \%)$, seguidas por artigos $(4 ; 40 \%)$ e tese $(1 ; 10 \%)$. Além disso, foi possível observar que o país de origem dos estudos foi predominantemente o Brasil (9; 90\%); com uma pesquisa proveniente do Chile (10\%) (Tabela 1).

Em relação à quantidade de autores, ela é superior à de estudos analisados devido a algumas pesquisas apresentarem mais de um pesquisador. Quanto à formação dos autores, identifica-se que a maior quantidade de profissionais foi da área de enfermagem (Tabela 1), aspecto que pode estar associado ao fato de essa classe de trabalhadores constituir maior número nos serviços de saúde, estar mais vinculada à prestação de assistência e desenvolver cada vez mais a atividade de preceptoria.
Tabela 1 - Caracterização das pesquisas analisadas.

\begin{tabular}{|c|c|c|}
\hline Variável & $\mathbf{N}$ & $\%$ \\
\hline \multicolumn{3}{|l|}{ Tipo de estudo } \\
\hline Dissertação & 5 & 50 \\
\hline Artigo & 4 & 40 \\
\hline Tese & 1 & 10 \\
\hline \multicolumn{3}{|l|}{ País } \\
\hline Brasil & 9 & 90 \\
\hline Chile & 1 & 10 \\
\hline \multicolumn{3}{|c|}{ Área de Formação do autor* } \\
\hline Não encontrado & 9 & 90 \\
\hline Enfermagem & 3 & 30 \\
\hline Pediatria & 2 & 20 \\
\hline Saúde da Família & 2 & 20 \\
\hline Odontologia & 1 & 10 \\
\hline Fisioterapia & 1 & 10 \\
\hline Filosofia & 1 & 10 \\
\hline Medicina & 1 & 10 \\
\hline Serviço social & 1 & 10 \\
\hline Ensino na saúde & 1 & 10 \\
\hline Saúde Coletiva & 1 & 10 \\
\hline Engenharia Elétrica & 1 & 10 \\
\hline Educação & 1 & 10 \\
\hline
\end{tabular}

Fonte: Autoria própria (2019).

Não obstante, ocorreu uma dificuldade na identificação da formação de alguns autores, devido a não ser apresentados durante a pesquisa ou não possuírem currículo lattes. Dessa forma, esses casos foram identificados como não encontrados (Tabela 1). Observa-se que a dimensão temporal variou de 2009 a 2018, ocorrendo uma produção ascendente ao longo dos anos de pesquisas sobre esse contexto na área da educação na saúde, com destaque dos anos de 2016 e 2018, ambos apresentando três publicações (30\%) (Figura 2). 
Figura 2 - Anos de publicação dos artigos analisados (em número absoluto).

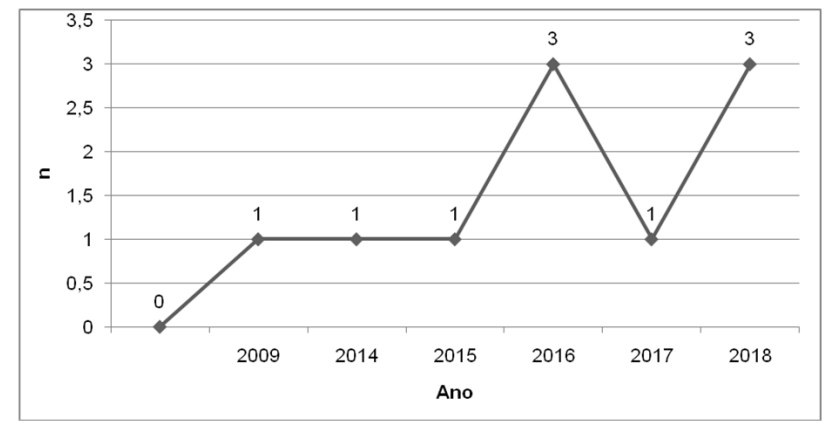

Fonte: elaborado pelos autores (2020).

Essa ascensão observada na Figura 2 pode ser explicada pela preocupação recente sobre o uso de metodologias ativas pelos preceptores, assim como necessária reflexão atual sobre suas contribuições e obstáculos. Quanto ao uso de metodologias ativas, foram citadas 13 diferentes metodologias, destacando-se suas potencialidades e desafios, conforme elucidado no Quadro 1.

Quadro 1 - Metodologias identificadas nos estudos analisados e suas respectivas potencialidades e desafios.

\begin{tabular}{|c|c|c|}
\hline Metodologias ativas (n) & Potencialidades & Desafios \\
\hline Tenda invertida (3) & $\begin{array}{l}\text { Espaços de trocas de } \\
\text { experiências } \\
\text {-Melhoria no processo de } \\
\text { trabalho em saúde }\end{array}$ & $\begin{array}{c}\text {-Falta de tempo devido à demanda } \\
\text {-Perda para o processo de formação } \\
\text { dos residentes }\end{array}$ \\
\hline Projeto Terapêutico Singular (PTS) (3) & $\begin{array}{l}\text {-Cuidado interprofissional } \\
\text {-Estímulo à autonomia e } \\
\text { cidadania entre os envolvidos } \\
\text {-Melhoria no processo de } \\
\text { trabalho em saúde } \\
\text {-Integralidade da atenção }\end{array}$ & $\begin{array}{c}\text {-Falta de tempo devido à demanda } \\
\text {-Realização de tarefas do serviço, } \\
\text { nas quais não podem ser realizados } \\
\text { questionamentos }\end{array}$ \\
\hline Roda de núcleo (2) & \multirow{4}{*}{$\begin{array}{l}\text {-Desenvolvimento de um } \\
\text { olhar multiprofissional e da } \\
\text { interprofissionalidade }\end{array}$} & \multirow{4}{*}{$\begin{array}{l}\text {-Dificuldade em compartilhar } \\
\text { conhecimento entre as categorias } \\
\text {-Dificuldade no acesso aos diversos } \\
\text { setores do hospital } \\
\text {-Não ser dada a devida importância } \\
\text { ao trabalho desempenhado }\end{array}$} \\
\hline Roda de campo (2) & & \\
\hline Módulos transversais (1) & & \\
\hline Módulos específicos (1) & & \\
\hline Educação interprofissional (2) & $\begin{array}{l}\text {-Desenvolvimento de um } \\
\text { olhar multiprofissional }\end{array}$ & \multirow{2}{*}{$\begin{array}{l}\text {-Falta de tempo devido à demanda } \\
\text {-Integração ensino-serviço } \\
\text {-Os servidores têm uma agenda } \\
\text { de trabalho inflexível e sem } \\
\text { planejamento } \\
\text {-Reconhecimento desse movimento e } \\
\text { seu fortalecimento }\end{array}$} \\
\hline Aprendizagem prática colaborativa (1) & $\begin{array}{l}\text {-Melhoria no processo de } \\
\text { trabalho em saúde }\end{array}$ & \\
\hline
\end{tabular}




\begin{tabular}{|c|c|c|}
\hline $\begin{array}{l}\text { Metodologia de aprendizagem baseada } \\
\text { em problemas (1) }\end{array}$ & $\begin{array}{c}\text {-Competências genéricas dos } \\
\text { futuros profissionais da saúde } \\
\text {-Favorecimento de um clima } \\
\text { de confiança } \\
\text {-Favorecimento do processo } \\
\text { de ensino-aprendizagem } \\
\text {-Entrega de valores e espaço } \\
\text { para críticas construtivas } \\
\text {-Aprendizagem refletindo } \\
\text { sobre sua própria ação } \\
\text {-Melhoria no processo de } \\
\text { trabalho em saúde }\end{array}$ & $\begin{array}{l}\text {-Dificuldade em superar a lógica } \\
\text { tradicional dos serviços } \\
\text {-Exigência de um novo papel no } \\
\text { processo de gestão para acadêmicos } \\
\text {-O sistema avaliativo é complexo e } \\
\text { adquire uma conotação diferente }\end{array}$ \\
\hline Roda de equipe (1) & $\begin{array}{l}\text {-Espaços de trocas de } \\
\text { experiências }\end{array}$ & $\begin{array}{c}\text { - Estranhamento no início do } \\
\text { processo }\end{array}$ \\
\hline Atendimento em conjunto (1) & $\begin{array}{l}\text {-Desenvolvimento de um } \\
\text { olhar multiprofissional }\end{array}$ & $\begin{array}{c}\text {-Dificuldade em superar a lógica } \\
\text { tradicional dos serviços } \\
\text {-Prejuízo à produtividade e ao } \\
\text { trabalho }\end{array}$ \\
\hline Atividades compartilhadas (1) & $\begin{array}{l}\text {-Desenvolvimento da } \\
\text { interprofissionalidade } \\
\text {-Espaços de trocas de } \\
\text { experiências }\end{array}$ & $\begin{array}{c}\text {-Carga horária dedicada às atividades } \\
\text { práticas } \\
\text {-Carga horária exigida não acontece } \\
\text { como desejada } \\
\text {-Dificuldade em superar a lógica } \\
\text { tradicional dos serviços } \\
\text {-Amplitude do programa }\end{array}$ \\
\hline
\end{tabular}

Fonte: elaborado pelos autores (2020).

É possível identificar a existência de um número superior de metodologias ativas elencadas no Quadro 1 em relação à quantidade de estudos analisados. Isso ocorreu em detrimento de algumas pesquisas utilizarem mais de um método durante o seu processo diagnóstico.

Evidenciou-se o uso da Tenda Invertida e do PTS, além da roda de núcleo, roda de campo e educação interprofissional. De maneira geral, destacam-se como potencialidades no uso de metodologias ativas de ensino nas RMS: o estímulo à troca de experiências; o favorecimento do cuidado interprofissional; a aprendizagem significativa a partir da reflexão-ação; e a melhoria no próprio processo de cuidar em saúde.

Por sua vez, denotaram-se como desafios: o escasso tempo destinado ao processo pedagógico pelo preceptor devido à demanda de atividades como profissionais de saúde em atuação; a dificuldade de superar a lógica tradicional de ensino e do serviço; e o despreparo pedagógico do preceptor.

\section{DISCUSSÃO}

É perceptível que há uma incipiência de artigos que abordam a temática investigada. Em decorrência disso, identificaram-se poucos estudos para o desenvolvimento desta pesquisa. Isso ocorre pelo fato de a RMS ser um programa relativamente recente, no qual ainda se tem uma preocupação embrionária acerca dos métodos de ensino utilizados nesse espaço de formação.

Com isso, foi possível averiguar que são poucas as capacitações que preceptores recebem, ocasionando uma divergência a respeito de quem deveria assumir esse papel, levando em conta que docentes estariam mais preparados didaticamente para desempenhá-lo. Nesse contexto, observa-se a importância do preparo pedagógico para os preceptores, devido a alguns não receberem, durante a sua formação inicial, um preparo para desempenhar a função de docência, ou seja, eles executam atividades para as quais não foram capacitados.

Em detrimento disso, a falta de embasamento pedagógico é uma das dificuldades enfrentadas pelos preceptores, pois eles 
não foram capacitados sobre como agir em algumas situações e não sabem se estão desenvolvendo métodos corretos. Dessa forma, não possuem entendimento necessário para aplicar as metodologias de forma eficiente e eficaz, influenciando diretamente na compreensão dos discentes. Portanto, compreende-se que a capacitação pedagógica permanente com um ensino crítico-reflexivo é primordial para garantir a união entre o mundo da educação e do trabalho para o preceptor, além de melhorar a qualidade de formação dos residentes (STEINBACH, 2015).

Com relação ao país de origem dos estudos, identificou-se que $90 \%$ das pesquisas são oriundas do Brasil, o que pode estar associado ao fato de as RMS, no contexto brasileiro, terem sido fortalecidas pelo SUS, pois, nesse sistema, existe o princípio da integralidade, que visa garantir a qualidade do atendimento por meio da integração das ações e áreas (BRASIL, 2020). Dessa forma, há uma predominância das RMS no âmbito dos hospitais públicos brasileiros, sobretudo hospitais de ensino que recebem alunos para realizar as atividades práticas e os estágios, com ênfase no desenvolvimento da interprofissionalidade. A interprofissionalidade se dá pelo fato de os profissionais organizarem, de forma conjunta, o objeto ou a problemática de maneira integralizada com métodos compartilhados, mantendo um nível de interdependência entre a equipe (SILVA, 2014).

No que diz respeito aos anos das publicações sobre essa temática, identifica-se que as pesquisas analisadas são recentes e de desenvolvimento ascendente ao longo do tempo, sendo perceptível que existe uma necessidade de que haja discussões acerca das metodologias ativas usadas pelos preceptores no contexto das RMS, pois é notório que as metodologias são fundamentais para um aprendizado eficaz.

À vista disso, as metodologias ativas são essenciais quando os profissionais utilizam a Educação Interprofissional (EIP) como eixo fundamental, a qual ocorre "quando dois ou mais profissionais aprendem um com o outro, a partir do outro e sobre o outro para melhorar a colaboração e a qualidade do cuidado". Além disso, proporcionam um aprendizado de forma conjunta com todos os profissionais da saúde, fomentando habilidades e atributos para um trabalho em conjunto, com interação, melhorando a colaboração e qualidade (CAIPE, 2002; REEVES, 2016).

No tocante às metodologias ativas identificadas nos estudos analisados, sobressaíram-se dois métodos: Tenda Invertida e Projeto Terapêutico Singular (PTS). Respectivamente, na tenda invertida, "o educador vai ao cotidiano de atuação do educando, conhece os desafios ali existentes e torna-os matéria-prima do processo de ensino, aprendizagem e reflexão" (ANDRADE et al., 2004, p. 35), também compreendido como "um momento de acompanhamento de um preceptor de campo, que se desloca até outro campo de estágio, para monitorar um residente de sua mesma categoria" (AGUIAR, 2018, p. 28). Já o PTS "consiste num conjunto de propostas de condutas terapêuticas articuladas, para um sujeito individual ou coletivo, resultado da discussão de uma equipe interdisciplinar" (BRASIL, 2007, p. 40).

Além disso, foram citadas outras metodologias como: EIP, Roda de Campo e Roda de Núcleo, as quais foram abordadas duas vezes. Sobre a Roda de Campo, estas são realizadas uma vez por semana, quando é lançada uma temática que, posteriormente, será discutida com, no máximo, nove residentes das várias áreas. Por sua vez, a Roda de Núcleo proporciona momentos de discussão e atuação de situações que acontecem no campo de estágio, com a participação de todos os residentes daquele setor, tendo como facilitador um preceptor de referência. Dessa forma, observa-se que o método da Roda pode ser conceituado como uma ferramenta que auxilia na construção da cogestão de coletivos (AGUIAR, 2018; CAMPOS, 2000). 
Não obstante, foi citada outra metodologia, a Rede de Saberes, um espaço quinzenal de produção coletiva e interdisciplinar de conhecimento, que acontece durante os dois anos da Residência, e que busca integrar todos os residentes, docentes, tutores, preceptores, representantes da gestão dos serviços de saúde e convidados. Apesar de ter sido citada apenas uma vez, essa metodologia apresenta potencialidades que garantem uma formação de qualidade (NASCIMENTO, 2016).

Considerando as metodologias ativas citadas pelas pesquisas analisadas, pode-se identificar como potencialidades: mais possibilidades detroca de experiências; melhoria do cuidado interprofissional; aprendizagem por intermédio do questionamento; e melhoria do processo de trabalho, favorecendo a interdisciplinaridade e a multiprofissionalidade.

Os métodos ativos utilizados foram eficientes e eficazes na promoção de aprendizagem ativa e interprofissional no âmbito das RMS. Um estudo realizado no Hospital Geral de Fortaleza (HGF), com os preceptores da residência multiprofissional em Neurologia e Neurocirurgia, com o objetivo de analisar a percepção dos preceptores acerca do processo de interdisciplinaridade e prática colaborativa, verificou a interdisciplinaridade como uma potencialidade proporcionada pelas metodologias ativas, sendo elucidado que esta é essencial porque vai capacitar o futuro profissional com uma visão ampla dentro de uma equipe multiprofissional (AGUIAR, 2018).

Já com relação à troca de experiências e à aprendizagem por intermédio do questionamento, pesquisadores relataram que os momentos da roda são muito importantes no contexto das RMS, como foi observado na pesquisa realizada no Programa de Residência Multiprofissional em Saúde da Família e Comunidade (PRMSFC) da Escola de Saúde Pública do Ceará (ESP/CE), com residentes, preceptores e coordenadores, objetivando analisar a sua proposta pedagógica. estudo apontou como falas dos entrevistados relatando estas potencialidades:

[...] a gente problematizava mesmo sobre o que a gente enfrentava em nosso cotidiano de trabalho e qual a melhor forma de a gente enfrentar isso [...] é uma atividade que eu percebo que facilita a colaboração [...] na roda de campo a gente consegue ver diferentes visões de uma mesmo situação e a gente consegue entender que eu posso somar com a visão do outro [...] (ARRUDA et al., 2016, p. 202).

Ademais, as potencialidades do PTS também foram enfatizadas por diferentes entrevistados:

[...] a partir do momento também que você estimula atividades como, por exemplo, a roda de equipe, o PTS [Projeto Terapêutico Singular], o apoio matricial, essas atividades você também consegue tá dando um incentivo pra colaboração interprofissional [...] eu acho que precisava ter espaços mais de roda mesmo, em campo, que proporcionasse construção de PTS. Para mim, esse é um espaço muito forte de troca [...] (ARRUDA et al., 2016, p. 197).

Com relação aos desafios, pode-se identificar: as dificuldades dos preceptores em destinar um tempo adequado para o ensino; bem como o desafio de modificar o ensino tradicional e a falta de capacitações para o preceptor. Apesar de apresentar muitos resultados benéficos, ainda é possível identificar dificuldades para aplicação de todos os métodos ativos abordados, pois a carga horária dos preceptores é dedicada às atividades práticas, o que foi elencado como um dos principais desafios, abordando ainda a dimensão atitudinal, pois, mesmo que essa formação garanta a interprofissionalidade, depende do preceptor e do residente dar continuidade a esses métodos. Nesse viés, 
é perceptível a necessidade de tempo para esses atores compreenderem as possibilidades de práticas colaborativas (ARRUDA et al., 2016; NASCIMENTO, 2016).

Outrossim, a atividade de preceptoria é adicionada a outras atividades das suas rotinas laborais, mas os seus atendimentos não são modificados, ou seja, é mais uma tarefa para ser realizada, que se torna difícil devido a sua demanda de trabalho. Também se identifica que os profissionais trabalham com uma agenda inflexível e, por vezes, sem planejamento pedagógico (NASCIMENTO, 2016). Pode-se verificar, assim, que a falta de tempo devido à demanda é um fator negativo na maioria das metodologias abordadas.

Além disso, outra dificuldade enfrentada é a superação da lógica tradicional dos serviços e da obrigatoriedade de tarefas no PTS, pois se trata de uma ferramenta de trabalho própria do serviço de saúde, ou seja, deve ser vista como uma atividade cotidiana e não apenas como uma tarefa extraordinária (ARRUDA et al., 2016). Outro fator identificado como dificultoso para aplicação das metodologias foi a falta de embasamento pedagógico durante a preceptoria, uma vez que os preceptores possuem dificuldade de agir em determinada situação e/ou sentem dúvida se estão agindo de forma correta.

Sobre isso, em pesquisa realizada na UBS do município de Florianópolis (SC), promovida pela Universidade Federal de Santa Catarina em parceria com a Secretaria Municipal de Saúde (SMS), com profissionais da saúde que atuam como preceptores, um dos preceptores relatou:

[...] Não me senti (professora), mas em algum momento tu tens que ser, e é isso que mais pega. Em que momentos? Como fazer isso? A nossa relação é muito horizontal, apesar que tem momentos que eu vou ter que ter outra postura. E que momento que vai ser esse? Como que vai ser isso? Então com o tempo a gente vai pegando essas manhas [...] (STEINBACH, 2015, p. 58).
Na mesma investigação, outro preceptor acrescentou: "eu dava conta, mas eu acho que tinha que avançar muito ainda. Porque era uma coisa muito empírica, sem embasamento, sem a parte pedagógica, formal, por isso a questão da capacitação" (STEINBACH, 2015, p. 58).

Tendo em vista as informações identificadas nos estudos, foi possível compreender que as potencialidades das metodologias ativas são inquestionáveis, a partir dos pontos positivos observados e dos benefícios garantidos. Apesar disso, ainda se nota a falta de capacitação pedagógica por parte dos preceptores, que é imprescindível que seja superada para, dessa forma, melhorar a qualidade de ensino da RMS.

\section{CONCLUSÃO}

As metodologias ativas de ensino têm sido utilizadas pelos preceptores nas RMS como método de ensino para promover um processo de ensino-aprendizagem dos profissionais em saúde ativo e colaborativo. Desse modo, é possível que tanto preceptores quanto docentes possam ser protagonistas, garantindo a interprofissionalidade, pois o conhecimento é desenvolvido por meio da troca entre os profissionais de forma participativa.

No tocante às limitações, verificou-se a escassez de estudos que abordam a temática em questão. Além disso, houve uma limitação identificada no Portal CAPES, pois este disponibilizava estudos na íntegra somente a partir do ano de 2013. Não obstante, muitas pesquisas questionavam apenas 0 conhecimento dos preceptores sobre as metodologias ativas, mas não realizavam a sua aplicação e, desse modo, não foram incluídas na amostra analisada. Os resultados apontados, portanto, devem ser interpretados a partir de tais limitações.

Por intermédio desta pesquisa, podem-se compreender quais metodologias ativas foram utilizadas no processo de formação 
dos residentes, podendo, dessa forma, verificar as suas potencialidades e os desafios, ou seja, identificar quais são os melhores métodos para serem aplicados e quais melhorias podem ser realizadas. Espera-se contribuir para a maior discussão da temática, incentivando a relação de pesquisas científicas que verifiquem o impacto do uso de metodologias ativas nas RMS. 


\section{REFERÊNCIAS}

AGUIAR, R.B.P.L. Interdisciplinaridade e prática colaborativa na percepção dos preceptores de um programa de residência multiprofissional em neurologia e neurocirurgia 2018. Dissertação (Mestrado) - Centro Universitário Christus, Fortaleza, 2018.

ANDRADE, L.O.M. et al. Escola de formação em saúde da família visconde de sabóia: três anos construindo a tenda invertida e a educação permanente no sus. Sanare, v. 5, n. 1, p. 1-7, 2004.

ARKSEY, H.; O'MALLEY, L. Scoping studies: towards a methodological framework. International Journal of Social Research Methodology, v. 8, n. 1, p. 19-32, 2005.

ARRUDA, G.M.M.S. et al. Educação interprofissional na pós-graduação em saúde: dimensões pedagógicas interprofissionais em uma Residência Multiprofissional em Saúde da Família. Tempus, actas de saúde colet, Brasília, v. 10, n. 4, p. 187-214, 2016.

BERBEL, N.A.N. As metodologias ativas e a promoção da autonomia de estudantes, Semina: Ciências Sociais e Humanas, v. 32, n. 1, p. 25-40, 2011.

BRASIL. Ministério da Educação. Residência Multiprofissional. 2018. Disponível em: http:// portal.mec.gov.br/residencias-em-saude/residencia-multiprofissional. Acesso em: 17 ago. 2020.

BRASIL. Ministério da Saúde, Secretaria de Atenção à Saúde, Núcleo Técnico da Política Nacional de Humanização. Clínica ampliada, equipe de referência e projeto terapêutico singular. Brasília: Ministério da Saúde, 2007.

BRASIL. Ministério da Saúde. Lei n 11.129, de 30 de junho de 2005. Institui a Residência em Área Profissional de Saúde e cria a Comissão Nacional de Residência Multiprofissional em Saúde CNRMS. Brasília: Ministério da Saúde, 2005.

BRASIL. Ministério da Saúde. Lei n 8.080, de 19 de setembro de 1990. Dispõe sobre as condições para a promoção, proteção e recuperação da saúde, a organização e o funcionamento dos serviços correspondentes e dá outras providências. Brasília: Ministério da Saúde, 1990.

BRASIL. Ministério da Saúde. Princípios do SUS. 2020. Disponível em: https://www.saude. gov.br/sistema-unico-de-saude/principios-do-sus. Acesso em: 17 ago. 2020.

BRASIL. Ministério da Saúde. Secretaria de Gestão do Trabalho e da Educação na Saúde. Residência multiprofissional em saúde: experiências, avanços e desafios. Brasília: Ministério da Saúde, 2006.

CAIPE. Centre for the advancement of interprofessional education. Interprofessional education: the definition. 2002. Disponível em: http://caipe.org.uk/resources/defining-ipe/. Acesso em: 17 ago. 2020. 
CAMPOS, G.W.S. Um método para análise e co-gestão de coletivos:

a constituição de sujeito, a produção de valor de uso e a democracia em instituições - o método da roda. 2000. Disponível em: https://www.gastaowagner. com.br/index.php?preview=1\&option=com_dropfiles\&format=\&task=frontfile. download\&catid=23\&id=74\&ltemid=1000000000000. Acesso em: 17 ago. 2020.

JOANNA BRIGGS INSTITUTE. The Joanna Briggs Institute Reviewer's Manual: methodology for JBI Scoping Reviews. South Australia (AU): The Joanna Briggs Institute, 2015.

LORENZ, R.H. Papel do preceptor na residência multiprofissional: experiência da fisioterapia. 2010. Dissertação (Mestrado) - Universidade Federal do Rio Grande do Sul, Porto Alegre, 2010.

MATTOS, T.M.C. Ideologia que permeia a prática da educação permanente por tutores e preceptores da residência multiprofissional em saúde. 2016. Tese (Doutorado) Universidade Federal do Rio de Janeiro, Rio de Janeiro, 2016.

NASCIMENTO, R.M.M. Educação interprofissional e preceptoria na residência multiprofissional em saúde da família. 2016. Dissertação (Mestrado) - Universidade Federal da Paraíba, João Pessoa, 2016.

REEVES, S. Porque precisamos da educação interprofissional para um cuidado efetivo e seguro. Interface, Botucatu, v. 20, n. 56, p. 185-196, 2016.

ROMAN, C. et al. Metodologias ativas de ensino-aprendizagem no processo de ensino em saúde no Brasil: uma revisão narrativa. Clinical and Biomedical Research, v. 37, n. 4, p. 349357, 2017.

SALVADOR, A.S. et al. Construindo a Multiprofissionalidade: um olhar sobre a Residência Multiprofissional em Saúde da Família e Comunidade. Revista Brasileira de Ciências da Saúde, v. 15, n. 3, p. 329-338, 2011.

SILVA, A.L.F. Programas de residência multiprofissional em saúde da família: repercussões no exercício profissional dos egressos de educação física. 2014. Dissertação (Mestrado) - Universidade Federal do Ceará, Sobral, 2014.

SILVA, C.T.D. et al. Residência Multiprofissional como espaço intercessor para a Educação Permanente em Saúde. Texto \& Contexto Enfermagem, v. 25, n. 1, e2760014, 2016.

STEINBACH, M.A. Preceptoria Na Residência Multiprofissional Em Saúde: saberes do Ensino e do Serviço. 2015. Dissertação (Mestrado) - Universidade Federal de Santa Catarina, Florianópolis, 2015.

TRICCO, A.C. et al. PRISMA. Extension for Scoping Reviews (PRIMA-SeR)- Checklist and Explanation. Annals of Internal Medicine, v. 169, n. 7, p. 467-473, 2018. 